\title{
Modelo Cinético Integrador para Fluxo de Gás Resultante do Movimento de Transferência de Massa em um Canal de Placas Heterogêneas
}

\author{
C. E. DA ROSA ${ }^{1 *}$,F. D. ROSSI ${ }^{2}$, L. F. JACOBI ${ }^{3}$ e R. F. KNACKFUSS ${ }^{4}$ \\ Recebido em 17 de janeiro de 2020 / Aceito em 10 de setembro de 2020
}

\begin{abstract}
RESUMO. Os fenômenos que envolvem a dinâmica de gases rarefeitos para fluxo de gases em microcanais têm sido fonte de estudo em várias pesquisas. A Equação de Boltzmann descreve tais fenômenos sendo que a partir da sua simplificação surge a teoria cinética para a dinâmica dos gases rarefeitos. Os modelos cinéticos apresentam resultados sólidos e é abordado, neste estudo, em uma versão analítica através do método de ordenadas discretas. Dessa maneira, exploramos um modelo mais próximo da realidade, comparando os valores numéricos gerados por quatro modelos cinéticos derivados de maneira unificada da Equação Linearizada de Boltzmann. Em termos de viscosidade e condutividade térmica os modelos são confrontados baseados na estatística não paramétrica para problemas da dinâmica de gases rarefeitos resultantes do movimento de transferência de massa. Além disso, utiliza-se a interação gás-superfície definida pelo núcleo de Cercignani-Lampis ao longo de um canal para placas heterogêneas.
\end{abstract}

Palavras-chave: dinâmica de gases rarefeitos, método de ordenadas discretas, modelos cinéticos, núcleo de Cercignani-Lampis.

\section{INTRODUÇÃO}

Os fenômenos que envolvem a dinâmica de gases rarefeitos para fluxo de gases em microcanais têm sido foco de muitos pesquisadores devido a inúmeras aplicações nesta área. Para descrever tais fenômenos, utiliza-se como base as Equações de Boltzmann (EB), que mesmo com a grande evolução computacional, ainda se mostra de difícil resolução, pois é uma equação diferencial parcial não linear com sete variáveis independentes e que contém um complexo operador

\footnotetext{
*Autor correspondente: Cinara Ewerling da Rosa - E-mail: cinara.rosa@iffarroupilha.edu.br

${ }^{1}$ Instituto Federal Farroupilha - Campus São Vicente do Sul, Universidade Federal de Santa Maria, Santa Maria-RS, Brasil - E-mail: cinara.rosa@iffarroupilha.edu.br https://orcid.org/0000-0002-9077-5031

2 Instituto Federal Farroupilha - Campus Alegrete, Alegrete-RS, Brasil - E-mail: fabio.rossi@iffarroupilha.edu.br https://orcid.org/0000-0002-2450-1024

${ }^{3}$ Universidade Federal de Santa Maria, Santa Maria-RS, Brasil - E-mail: lucianefj8@ gmail.com https://orcid.org/00000003-4622-9292

${ }^{4}$ Universidade Federal de Santa Maria, Santa Maria-RS, Brasil - E-mail: rfknackfuss@ gmail.com https://orcid.org/ 0000-0001-6997-3474
} 
de colisão. Nesse sentido, a fim de possibilitar o trabalho com a equação, foram desenvolvidos métodos de simplificação, nos quais, inicialmente visam uma versão linearizada. Mas, o trabalho com a Equação Linearizada de Boltzmann (ELB), conhecida como equação do transporte integro diferencial, ainda é muito restrito devido ao seu núcleo sintético visto que simulações numéricas na área de termodinâmica $[4,11]$, por exemplo, somente apresentaram resultados aceitáveis recentemente apesar dos esforços aplicados.

Diversos estudos [1,28,29,32,39] foram desenvolvidos visando uma simplificação da equação integro diferencial e mais recentemente com ênfase na utilização de gases nobres e suas aplicações $[17,18,35,38]$. Mas para isso, é necessário manter as características matemáticas e físicas fundamentais da EB [23]. Dessa maneira, foram definidas diversas equações modelo, onde o termo integral de colisão é simplificado, e entre esses os modelo estudados neste trabalho, são: Modelo BGK proposto por Bhatnagar, Gross e Krook [5], Modelo S proposto por Shakhov [27,30], Modelo Gross-Jackson (Modelo GJ) proposto por Gross e Jackson [15] e abordado por Scherer [25], Modelo MRS proposto por Garcia e Siewert [13]. A maioria dos trabalhos que abordam os modelos cinéticos apresentam um foco individual, ou seja, estudam equações modelo separadamente, com prioridade ao Modelo BGK, modelo mais simples [6, 18, 20]. Para uma melhor metodologia de estudo, propõe-se neste trabalho uma alternativa de descrever quatro modelos cinéticos unificadamente, proposto inicialmente por Garcia (2006) [13] para três modelos. Essa proposta define um núcleo de espalhamento geral que possui parâmetros que, conforme sua escolha, descrevem um modelo cinético em particular. Dessa maneira, tem-se a possibilidade de a partir de um único desenvolvimento analítico mais elaborado, reproduzir um maior número de resultados numéricos, visando assim, comparações e análises entre os modelos.

Para termos a possibilidade de reproduzir resultados numéricos de grandezas físicas de interesse, inicialmente deve-se definir um canal por onde irá ocorrer o fluxo de gás, que neste estudo será caracterizado por duas placas paralelas e não simétricas. Assim, é necessário observar que a escolha dessa superfície irá interferir no fluxo gasoso, dessa maneira, deve-se considerar ainda as condições de contorno para caracterizar esta ação juntamente com a EB simplificada. A condição de contorno mais utilizada atualmente é a de Maxwell, pela sua simplicidade. Contudo, esta condição de contorno não representa condições reais totalmente legítimas, pois possui apenas um coeficiente de acomodação, ou seja, define apenas uma propriedade física do gás. No entanto, o gás possui mais de uma propriedade física, isto é, necessita-se uma condição de contorno que ofereça a possibilidade de utilizarmos mais de uma propriedade do gás. Em busca de uma interação do gás com a superfície mais voltada para a realidade, utiliza-se as Condições de Contorno de Cercignani-Lampis (CL) [10]. Essas condições de fronteira são descritas como generalizadas, já que abrange dois coeficientes de acomodação que podem descrever até duas propriedades físicas do gás, ou seja, permite uma melhor descrição física para fenômenos de transporte de gases.

Por fim, para concluir o problema do nosso estudo, ainda é necessário definir o que irá provocar o movimento do gás que serão representados na parte não homogênea da equação íntegrodiferencial ou da Condição de Contorno de CL. Nesse sentido, serão abordados três proble- 
mas clássicos da dinâmica de gases rarefeitos: Poiseuille, Creep Térmico e Couette. A partir da definição do problema, a metodologia escolhida para resolução foi o método de ordenadas discretas (ADO), que deriva do modelo proposto por Wick G.C.(1984) [36], o qual difere pelo esquema de quadratura do tipo half-range, além da determinação das constantes de separação a partir da resolução de um problema de autovalores.

Nesse sentido o presente trabalho tem como objetivo apresentar uma proposta de solução unificada para Modelos cinéticos derivados da ELB através de placas paralelas heterogêneas. O método proposto é capaz de gerar resultados para vários modelos, de forma simplificada e com resultado satisfatório ao considerar equiparidade numérica quando comparado a ELB. Tais resultados numéricos são confrontados estatisticamente afim de encontrar o modelo com solução mais adequada com base na convergência dos dados de forma não paramétrica, em termos de qualidade do resultado e tempo de execução para os problemas de Poiseuille, Creep-Térmico e Couette.

\section{FORMULAÇÃO DO MODELO}

\subsection{Modelo Cinético}

Devido a ELB possuir uma estrutura ainda muito complexa de se trabalhar [8], uma alternativa para desmembrar esta equação é fazer uso das equações modelo, ou seja, equações cinéticas que definem uma expressão simplificada para desenvolver o núcleo de espalhamento da ELB em termos de uma perturbação, sem perder as características físicas do processo de colisão, e podem ser agrupadas considerando a frequência de colisão.

Neste trabalho, são desenvolvidos modelos definidas com frequência de colisão constante, com origem da ELB e que derivam da equação cinética para um canal plano da seguinte forma [3,37]:

$$
c_{x} \frac{\partial}{\partial \tau} h(\tau, \mathbf{c})+\varepsilon h(\tau, \mathbf{c})=\varepsilon \pi^{-3 / 2}+\int_{-\infty}^{\infty} \int_{-\infty}^{\infty} \int_{-\infty}^{\infty} e^{c^{\prime 2}} h\left(\tau, \mathbf{c}^{\prime}\right) F\left(\mathbf{c}^{\prime}: \mathbf{c}\right) d c_{x}^{\prime} d c_{y}^{\prime} d c_{z}^{\prime}+S(\mathbf{c})
$$

sendo a função $h(\tau, \mathbf{c})$ a perturbação causada por situações fracamente fora de equilíbrio, $c$ e $c^{\prime}$ variáveis adimensionais da velocidade derivado da linearização da $\mathbf{E B}, \mathbf{c}^{\prime}=\left(c_{x}^{\prime}, c_{y}^{\prime}, c_{z}^{\prime}\right)$ e $\mathbf{c}=$ $\left(c_{x}, c_{y}, c_{z}\right)$ as velocidades, ambas respectivamente, antes e depois da colisão.

Para introduzir a questão de adimensionalização da ELB, usa-se o livre caminho médio $(\mu)$, que é a distância percorrida por uma partícula sem sofrer colisão [12]. Logo, define-se a variável adimensional $\tau=x^{*} / \mu$ e o parâmetro $\varepsilon=\tau_{0}^{2} n_{0} \pi^{1 / 2} \mu$ onde $x^{*}$ é a variável espacial e $n_{0}$ é a densidade de equilíbrio das partículas de um gás. Segundo [3], após algumas manipulações algébricas, pode-se assim, calcular o livre caminho médio de duas maneiras, em termos de viscosidade ( $\left.\varepsilon=\varepsilon_{p}\right)$ e condutividade térmica $\left(\varepsilon=\varepsilon_{t}\right)$, tendo como base os estudos de [22] e [21], nessa ordem.

Além disso, o núcleo de espalhamento é dado por

$$
F\left(\mathbf{c}^{\prime}: \mathbf{c}\right)=1+2\left(\mathbf{c}^{\prime} \cdot \mathbf{c}\right)+\frac{2}{3}\left(c^{\prime 2}-\frac{3}{2}\right)\left(c^{2}-\frac{3}{2}\right)+\beta M\left(\mathbf{c}^{\prime}: \mathbf{c}\right)+\varpi N\left(\mathbf{c}^{\prime}: \mathbf{c}\right)
$$


com

$$
M\left(\mathbf{c}^{\prime}: \mathbf{c}\right)=4 / 5\left(\mathbf{c}^{\prime} \cdot \mathbf{c}\right)\left(c^{\prime 2}-5 / 2\right)\left(c^{2}-5 / 2\right)
$$

e

$$
N\left(\mathbf{c}^{\prime}: \mathbf{c}\right)=2\left[\left(\mathbf{c}^{\prime} \cdot \mathbf{c}\right)^{2}-1 / 3 c^{\prime 2} c^{2}\right]
$$

Define-se ainda, o termo de fonte, dado por

$$
S(\mathbf{c})=-c_{y}\left(k_{P}+k_{T}\left(c_{x}^{2}+c_{y}^{2}+c_{z}^{2}-5 / 2\right)\right)
$$

sendo que $k_{P}$ e $k_{T}$ são os gradientes constantes de pressão e temperatura respectivamente.

Serão desenvolvidas, neste estudo, as equações modelo definidos como Modelo BGK [5], Modelo S [27, 30], Modelo GJ [15] e Modelo MRS [13]. Segundo Garcia [13], para descrever matematicamente cada modelo cinético considera-se os parâmetros apresentados na Tabela 1.

Tabela 1: Valores dos parâmetros para cada equação modelo.

\begin{tabular}{lcccc}
\hline Modelo Cinético & $\beta$ & $\varpi$ & $\varepsilon_{p}$ & $\varepsilon_{t}$ \\
\hline Modelo BGK & 0 & 0 & 1 & 1 \\
Modelo S & $1 / 3$ & 0 & 1 & $3 / 2$ \\
Modelo GJ & $5 / 9$ & $1 / 3$ & $3 / 2$ & $9 / 4$ \\
Modelo MRS & $1-(16 / 15) 2^{1 / 2}$ & $1-(8 / 5) 2^{1 / 2}$ & $(5 / 16) 2^{1 / 2}$ & $(15 / 32) 2^{1 / 2}$ \\
\hline
\end{tabular}

\subsection{Condição de Contorno}

O modelo de CL [31] é utilizado no processo de interação entre o gás e a parede que é definido em termos dos coeficientes de acomodação normal e tangencial. Geralmente, a função de distribuição é definida pela função de distribuição relativa $f\left(\mathbf{v}^{\prime}\right)$ e pelo núcleo de espalhamento $R\left(\mathbf{v}^{\prime}: \mathbf{v}\right)$ [9], que delimita a forma de interação que ocorre entre o gás e a superfície sólida e é expresso por

$$
\left|v_{n}\right| f(\mathbf{v})=\int_{v_{n}^{\prime}<0}\left|v_{n}^{\prime}\right| R\left(\mathbf{v}^{\prime}: \mathbf{v}\right) f\left(\mathbf{v}^{\prime}\right) d \mathbf{v}^{\prime}, \quad v_{n}>0
$$

onde as variáveis ilustradas na Figura 1 são denominadas como velocidade molecular da partícula de incidência $\left(\mathbf{v}^{\prime}\right)$, vetor unitário normal à superfície $(\mathbf{n})$ e componente normal da velocidade $\left(v_{n}\right)$, ou seja, $v_{n}=\mathbf{v} \cdot \mathbf{n}$.

Além disso, de acordo com Cercignani [9], o núcleo de espalhamento precisa satisfazer a condição de normalização, não-negatividade e relação de reciprocidade. 


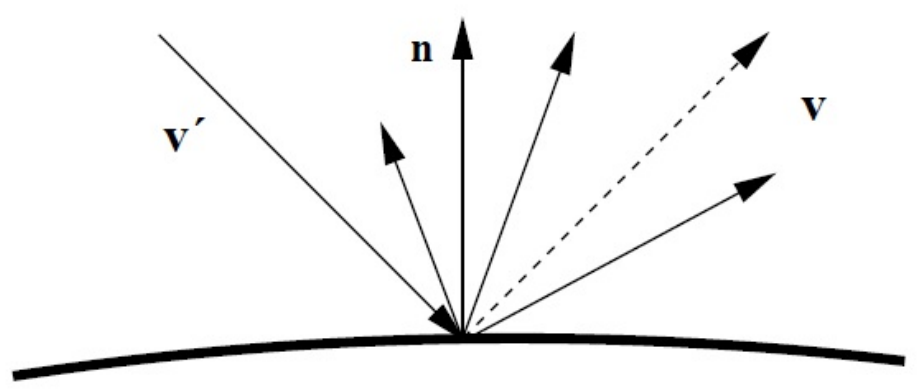

Figura 1: Interação gás-superfície [16].

O núcleo de $\mathbf{C L}$ para coordenadas retangulares, em termos da velocidade adimensional $(c=$ $\left.v\left[m /\left(2 k_{B} T_{0}\right)\right]^{1 / 2}\right)$ derivado da linearização da $\mathbf{E B}$ é dado por

$$
\begin{aligned}
R_{l}\left(\mathbf{v}^{\prime}: \mathbf{v}\right)= & \frac{m^{2} v_{n}}{2 \pi \alpha_{n_{l}} \alpha_{t_{l}}\left(2-\alpha_{t_{l}}\right)\left(k_{B} T_{w}\right)^{2}} \widehat{I}_{0}\left(\frac{\left(1-\alpha_{n_{l}}\right)^{1 / 2} m v_{n} v_{n}^{\prime}}{\alpha_{n_{l}} k_{B} T_{w}}\right) \\
& \exp \left[-\frac{m\left[v_{n}^{2}+\left(1-\alpha_{n_{l}}\right) v_{n}^{\prime 2}\right]}{2 k_{B} T_{w} \alpha_{n_{l}}}-\frac{m\left[\mathbf{v}_{t}-\left(1-\alpha_{t_{l}}\right) \mathbf{v}_{t}^{\prime}\right]^{2}}{2 k_{B} T_{w} \alpha_{t_{l}}\left(2-\alpha_{t_{l}}\right)}\right]
\end{aligned}
$$

sendo $\mathbf{v}_{t}$ o vetor bidimensional de velocidade tangencial e $\mathbf{v}_{n}$ a componente normal da velocidade $\mathbf{v}, m$ a massa molecular, $k_{B}$ a constante de Boltzmann e $T_{0}$ a temperatura constante de referência. Além disso, $l=1,2$ define as placas inferior e superior do canal. É possível observar que o núcleo de $\mathbf{C L}$ pode representar placas heterogêneas, ou seja, paredes da superfície com propriedades físicas diferentes.

Além disso, para fins computacionais utiliza-se uma função de Bessel modificada definida como

$$
\widehat{I_{0}}(w)=\frac{e^{-w}}{2 \pi} \int_{0}^{2 \pi} e^{w \cos \Phi} d \Phi .
$$

Nota-se que o núcleo dado pela Eq. 2.2 possui dois parâmetros que segundo Sharipov e Seleznev [30] são definidos como coeficiente de acomodação tangencial em $0 \leq \alpha_{t_{l}} \leq 2$ e coeficiente de acomodação da energia cinética devido a componente normal da velocidade em $0 \leq \alpha_{n_{l}} \leq 1$.

Salienta-se que o núcleo de CL pode ser considerado como um caso geral do núcleo difusoespecular, ou seja, assumindo $\alpha_{t_{l}}=\alpha_{n_{l}}=1$, o núcleo torna-se difuso e quando $\alpha_{t_{l}}=\alpha_{n_{l}}=0$, o núcleo coincide com o núcleo especular [28].

A condição de contorno cujo o núcleo de espalhamento é proposto por $\mathbf{C L}$ dado pela Eq. 2.2, linearizada e adimensional para o vetor velocidade $(c)$ escreve-se em coordenadas retangulares para placas diferentes [34] como

$$
\begin{aligned}
h\left(\mp a, \pm c_{x}, c_{y}, c_{z}\right)= & 2 u_{w_{l}} \alpha_{t_{l}} c_{y}+\int_{0}^{\infty} \int_{-\infty}^{\infty} \int_{-\infty}^{\infty} h\left(\mp a, \mp c_{x}^{\prime}, c_{y}^{\prime}, c_{z}^{\prime}\right) \\
& R_{l}\left(\mp c_{x}^{\prime}, c_{y}^{\prime}, c_{z}^{\prime}: \pm c_{x}, c_{y}, c_{z}\right) d c_{x}^{\prime} d c_{y}^{\prime} d c_{z}^{\prime}
\end{aligned}
$$


onde

$$
R_{l}\left(c_{x}^{\prime}, c_{y}^{\prime}, c_{z}^{\prime}: c_{x}, c_{y}, c_{z}\right)=\frac{2 c_{x}^{\prime}}{\pi \alpha_{n_{l}} \alpha_{t_{l}}\left(2-\alpha_{t_{l}}\right)} T\left(c_{y}^{\prime}: c_{y}\right) S\left(c_{x}^{\prime}: c_{x}\right) T\left(c_{z}^{\prime}: c_{z}\right)
$$

com

$$
T(x: z)=\exp \left[\frac{-\left[\left(1-\alpha_{t_{l}}\right) z-x\right]^{2}}{\alpha_{t_{l}}\left(2-\alpha_{t_{l}}\right)}\right]
$$

$\mathrm{e}$

$$
S(x: z)=\exp \left[\frac{-\left[\left(1-\alpha_{n_{l}}\right)^{1 / 2} z-x\right]}{\alpha_{n_{l}}}\right] \widehat{I_{0}}\left[\frac{2\left(1-\alpha_{n_{l}}\right)^{1 / 2}|x z|}{\alpha_{n_{l}}}\right]
$$

para $c_{x} \in(0, \infty), c_{y}, c_{z} \in(-\infty, \infty)$ e $\widehat{I}_{0}$ dado pela Eq. 2.3. Além disso, o termo não homogêneo da condição de contorno é dado pelo parâmetro $u_{w_{l}}$ para $l=1,2$ que representa a velocidade da placa inferior e superior.

\subsection{Problemas da dinâmica de gases rarefeitos}

Conforme Figura 2 é mostrado os problemas da dinâmica de gases rarefeito para transferência de massa entre placas heterogêneas. Considera-se dois reservatórios contendo o mesmo gás rarefeito interligados por duas placas paralelas de comprimento $l$ e largura $x \in[-a, a]$ tal que $l \gg a$, não necessariamente compostas pela mesma composição química. As paredes estão separadas por uma distância $2 a$, localizadas em $\tau=a$ e $\tau=-a$, no qual, o gás está fluindo na direção $y$. O fluxo do gás dentro dos reservatórios ocorre devido a uma pressão $\left(P_{i}\right)$, concentração molar $\left(C_{i}\right)$ e temperatura $\left(T_{i}\right)$, para $i=1,2$, sendo que $P_{1}>P_{2}, C_{1}>C_{2}$ e $T_{1}>T_{2}$, para que ocorra movimento do gás (apenas na direção y paralela as placas). Ainda, pode ocorrer fluxo de gás a partir do movimento das placas. Nota-se que, devido ao gradiente de pressão $\left(k_{P}\right)$, temperatura $\left(k_{T}\right)$ ou velocidade das placas $\left(u_{w}\right)$ ocorre fenômenos de transferência de massa, definidos no modelo cinético e condição de contorno através do termo não-homogêneo. Estaremos dando ênfase aos seguintes problemas: Poiseuille, Creep Térmico e Couette.

Definido os problemas de dinâmica de gases rarefeitos é possível definir as quantidades físicas de interesse, que neste caso será o perfil de velocidade e perfil fluxo de calor.

A formulação analítica do perfil de velocidade [31] é dada por

$$
u(\tau)=\frac{1}{\pi^{3 / 2}} \int_{-\infty}^{\infty} \int_{-\infty}^{\infty} \int_{-\infty}^{\infty} e^{-\mathbf{c}^{\prime 2}} h\left(\tau, c_{x}, c_{y}, c_{z}\right) c_{x} d c_{x} d c_{y} d c_{z}
$$

e o perfil fluxo de calor [31] dado pela seguinte expressão

$$
q(\tau)=\frac{1}{\pi^{3 / 2}} \int_{-\infty}^{\infty} \int_{-\infty}^{\infty} \int_{-\infty}^{\infty} e^{-\mathbf{c}^{\prime 2}}\left(\mathbf{c}^{2}-\frac{5}{2}\right) h\left(\tau, c_{x}, c_{y}, c_{z}\right) c_{x} d c_{x} d c_{y} d c_{z} .
$$




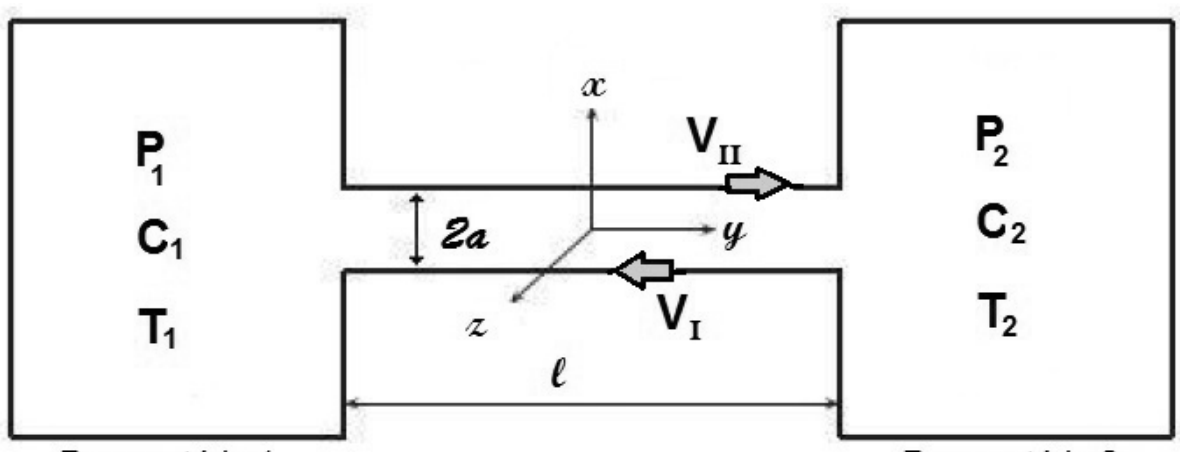

Reservatório 1

Reservatório 2

Figura 2: Escoamento de um gás em um canal plano.

\subsection{Formulação Vetorial}

Ao analisar as equações que descrevem as grandezas físicas de interesse nota-se que são definidas a partir de momentos da função $h(\tau, \mathbf{c})$. Além disso, deve-se escrever a ELB em uma forma vetorial utilizando equações auxiliares para resolução do problema original. Dessa maneira, baseado nos trabalhos [19] e [18] define-se as seguintes equações auxiliares:

$$
h_{1}\left(\tau, c_{x}\right)=\int_{-\infty}^{\infty} \int_{-\infty}^{\infty} c_{y} \pi^{-1} e^{-\left(c_{y}^{2}+c_{z}^{2}\right)} h\left(\tau, c_{x}, c_{y}, c_{z}\right) d c_{y} d c_{z}
$$

$\mathrm{e}$

$$
h_{2}\left(\tau, c_{x}\right)=\int_{-\infty}^{\infty} \int_{-\infty}^{\infty} 2^{-1 / 2} \pi c_{y}\left(c_{y}^{2}+c_{z}^{2}-2\right) e^{-\left(c_{y}^{2}+c_{z}^{2}\right)} h\left(\tau, c_{x}, c_{y}, c_{z}\right) d c_{y} d c_{z}
$$

A partir de manipulações algébricas na Eq. 2.1 e assumindo $c_{x}=\xi$, obtém-se a equação vetorial

$$
\xi \frac{\partial}{\partial \tau} \mathbf{H}(\tau, \xi)+\varepsilon \mathbf{H}(\tau, \xi)=\varepsilon \int_{-\infty}^{\infty} \Psi\left(\xi^{\prime}\right) \mathbf{K}\left(\xi^{\prime}, \xi\right) \mathbf{H}\left(\tau, \xi^{\prime}\right) d \xi^{\prime}+\mathbf{S}(\xi)
$$

em que,

$$
\begin{gathered}
\Psi(\xi)=\pi^{-1 / 2} e^{-\xi^{2}} \\
\mathbf{H}(\tau, \xi)=\left[\begin{array}{ll}
h_{1}(\tau, \xi) & h_{2}(\tau, \xi)
\end{array}\right]^{T}, \\
\mathbf{K}\left(\xi^{\prime}, \xi\right)=\left[\begin{array}{cc}
1+(2 \beta / 5)\left(\xi^{2}-1 / 2\right)\left(\xi^{\prime 2}-1 / 2\right)+2 \varpi \xi^{\prime} \xi & \left(2^{3 / 2} \beta / 5\right)\left(\xi^{2}-1 / 2\right) \\
\left(2^{3 / 2} \beta / 5\right)\left(\xi^{\prime 2}-1 / 2\right) & 4 \beta / 5
\end{array}\right]
\end{gathered}
$$

$\mathrm{e}$

$$
\mathbf{S}(\xi)=-k_{P}\left[\begin{array}{ll}
1 / 2 & 0
\end{array}\right]^{T}-\frac{k_{T}}{2}\left[\begin{array}{ll}
\xi^{2}-1 / 2 & 2^{1 / 2}
\end{array}\right]^{T} .
$$


Além disso, de maneira análoga as condições de contorno são reescritas baseadas na Eq. 2.4 na forma

$$
\mathbf{H}(\mp a, \pm \xi)=\alpha_{t_{l}} u_{w_{l}}\left[\begin{array}{ll}
1 & 0
\end{array}\right]^{T}+\mathbf{A}_{l} \int_{0}^{\infty} \mathbf{H}\left(\mp a, \mp \xi^{\prime}\right) f_{l}\left(\xi^{\prime}, \xi\right) d \xi^{\prime}
$$

com

$$
f_{l}\left(\xi^{\prime}, \xi\right)=\frac{2 \xi^{\prime}}{\alpha_{n_{l}}} \exp \left[\frac{-\left[\left(1-\alpha_{n_{l}}\right)^{1 / 2} \xi-\xi^{\prime}\right]^{2}}{\alpha_{n_{l}}}\right] \widehat{I_{0}}\left[\frac{2\left(1-\alpha_{n_{l}}\right)^{1 / 2} \xi \xi^{\prime}}{\alpha_{n_{l}}}\right]
$$

e

$$
\mathbf{A}_{l}=\left[\begin{array}{cc}
\left(1-\alpha_{t_{l}}\right) & 0 \\
0 & \left(1-\alpha_{t_{l}}\right)^{3}
\end{array}\right]
$$

para $\xi, \xi^{\prime} \in(0, \infty), l=1,2$ e $\widehat{I}_{0}$ dado pela Eq. 2.3.

Ainda, das Eqs.2.5 e 2.6, as quantidades físicas de interesse, em termos da solução $\mathbf{H}$, para o perfil de velocidade e fluxo de calor são dadas por

$$
u(\tau)=\int_{-\infty}^{\infty} \Psi(\xi)\left[\begin{array}{ll}
1 & 0
\end{array}\right] \mathbf{H}(\tau, \xi) d \xi
$$

e

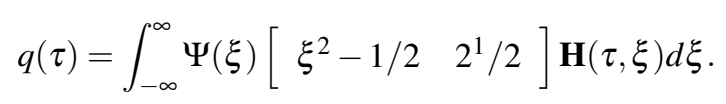

\subsection{Solução em Ordenadas Discretas}

A princípio será manipulado a forma vetorial (Eq. 2.7) da equação cinética (Eq. 2.1). A solução do problema $\mathbf{H}(\tau, \xi)$ é dado a partir da soma de uma solução homogênea $\mathbf{H}^{h}(\tau, \xi)$ com a solução particular $\mathbf{H}^{p}(\tau, \xi)$ a partir do método ADO.

Inicialmente, trabalha-se com a solução homogênea. Para isso, a partir da Eq. 2.7 é definido um esquema de quadratura $\operatorname{com} \xi= \pm \xi_{i}$ para $i=1, \ldots, N$, obtendo a seguinte equação:

$$
\begin{gathered}
\pm \xi_{i} \frac{\partial}{\partial \tau} \mathbf{H}^{h}\left(\tau, \pm \xi_{i}\right)+\varepsilon \mathbf{H}^{h}\left(\tau, \pm \xi_{i}\right)=\underset{k=1}{\varepsilon} \sum_{k}^{N} w_{k} \Psi\left(\xi_{k}\right)\left[\mathbf{K}\left(\xi_{k}, \pm \xi_{i}\right) \mathbf{H}^{h}\left(\tau, \xi_{k}\right)+\right. \\
\left.\mathbf{K}\left(-\xi_{k}, \mp \xi_{i}\right) \mathbf{H}^{h}\left(\tau,-\xi_{k}\right)\right]
\end{gathered}
$$

onde $\xi_{k}$ e $w_{k}$ são os conjuntos dos $N$ pontos de quadratura e pesos para $[0, \infty)$.

Propõe-se então, soluções exponenciais da forma $\mathbf{H}^{h}(\tau, \xi)=\Phi(v, \xi) e^{-\varepsilon \tau / v}$ onde o vetor $\Phi\left(v_{j}, \pm \xi_{k}\right)$ possui dimensão $2 N \times 1$, com $N$ componentes $2 \times 1$ redefinidas por $\Phi_{ \pm}\left(v_{j}\right)$ ao considerar a simetria de $\xi$ e $\xi^{\prime}$ para a matriz $\mathbf{K}\left(\xi^{\prime}, \xi\right)$.

A partir de manipulações algébricas detalhadas em [24], obtém-se um problema de autovalores e autovetores onde as constantes de separação são dadas por $v$. Portanto, a solução para a equação homogênea, usando o princípio da superposição é dada por

$$
\mathbf{H}_{ \pm}^{h}(\tau)=\sum_{j=1}^{2 N}\left[A_{j} \Phi_{ \pm}\left(v_{j}\right) e^{-\varepsilon(a+\tau) / v_{j}}+B_{j} \Phi_{\mp}\left(v_{j}\right) e^{-\varepsilon(a-\tau) / v_{j}}\right] .
$$


Além disso, $A_{j}$ e $B_{j}$ são os coeficientes de superposição, as chamadas constantes arbitrárias. Outrossim, os problemas de gases rarefeitos são conservativos [4], logo, um dos autovalores tende a zero quando $N$ tende ao infinito. Dessa forma, negligencia-se a constante de separação entre as $v_{j}$ de maior valor e reescreve-se a Eq. 2.12 como

$$
\mathbf{H}_{ \pm}^{h}(\tau)=A_{1} \Phi^{1}+B_{1} \Phi_{ \pm}^{2}(\tau)+\sum_{j=2}^{2 N}\left[A_{j} \Phi_{ \pm}\left(v_{j}\right) e^{\frac{-\varepsilon(a+\tau)}{v_{j}}}+B_{j} \Phi_{\mp}\left(v_{j}\right) e^{\frac{-\varepsilon(a-\tau)}{v_{j}}}\right]
$$

onde $\Phi^{1}$ e $\Phi_{ \pm}^{2}(\tau)$ são soluções exatas de dimensão $2 N \times 1$ definidas por um vetor de $N$ componentes da seguinte forma

$$
\Phi^{1}=\left[\begin{array}{ll}
1 & 0
\end{array}\right]^{T} \text { e } \Phi_{ \pm}^{2}(\tau)=\left[\varepsilon \tau \mp\left(\frac{\xi}{1-\bar{\omega}}\right) \quad 0\right]^{T}
$$

A fim de resolver o problema, ainda faz-se necessário desenvolver a solução particular descrita da seguinte forma:

$$
\mathbf{H}^{p}(\tau, \xi)=\mathbf{B} \tau^{2}+\mathbf{C} \tau \xi+\mathbf{D} \xi^{2}+\mathbf{E} \xi+\mathbf{F}
$$

em que B, C, D, E e $\mathbf{F}$ são vetores $2 \times 1$ com componentes constantes.

Substituindo-se então a Eq. 2.14 na Eq. 2.7 e usando a Eq. 2.8 tem-se a solução particular dada por

$$
\mathbf{H}^{p}(\tau, \xi)=k_{P}\left[\begin{array}{ll}
\varepsilon \frac{(1-\varpi) \tau^{2}}{2}-\tau \varepsilon+\frac{(5-4 \beta) \xi^{2}}{5 \varepsilon(1-\beta)} & \frac{2^{1 / 2} \beta}{5 \varepsilon(1-\beta)}
\end{array}\right]^{T}+\frac{k_{T}}{2 \varepsilon(\beta-1)}\left[\begin{array}{ll}
\xi^{2} & 2^{1 / 2}
\end{array}\right]^{T} .
$$

Assumindo para o Problema de Poiseuille $k_{P}=1$ e $k_{T}=0$, problema Creep Térmico, $k_{P}=0$ e $k_{T}=1$. Note que para o Problema de Couette a solução particular é nula e portanto temos que $\mathbf{H}^{h}(\tau, \xi)=\mathbf{H}(\tau, \xi)$.

Portanto, encontrada a solução homogênea e particular do problema pode-se escrever a solução geral como

$$
\begin{aligned}
\mathbf{H}_{ \pm}(\tau)= & \mathbf{H}_{ \pm}^{p}(\tau)+A_{1} \Phi^{1}+B_{1} \Phi_{ \pm}^{2}(\tau)+ \\
& \sum_{j=2}^{2 N}\left[A_{j} \Phi_{ \pm}\left(v_{j}\right) e^{-\varepsilon(a+\tau) / v_{j}}+B_{j} \Phi_{\mp}\left(v_{j}\right) e^{-\varepsilon(a-\tau) / v_{j}}\right]
\end{aligned}
$$

Ainda, partindo da condição de contorno dada pela Eq. 2.9, define-se um esquema de quadratura para o intervalo $[0, \infty) \operatorname{com} \xi= \pm \xi_{i}$. Após discretizar a integral tem-se:

$$
\mathbf{H}\left(\mp a, \pm \xi_{i}\right)=\mathbf{A}_{l} \sum_{k=1}^{N} w_{k} \mathbf{H}\left(\mp a, \mp \xi_{k}\right) f_{l}\left(\xi_{k}, \pm \xi_{i}\right)+\alpha_{t_{l}} u_{w_{l}}\left[\begin{array}{ll}
1 & 0
\end{array}\right]^{T}
$$

onde $\xi_{k}$ e $w_{k}$ são os conjuntos dos $N$ pontos de quadratura e $N$ pesos obtidos para o intervalo $[0, \infty)$, para $l=1,2$ com $f_{l}\left(\xi^{\prime}, \xi\right)$ e $\mathbf{A}_{l}$ dado pelas Eqs. 2.10 e 2.11, respectivamente. Salienta-se que a solução particular da condição de contorno de CL é dada por:

$$
\mathbf{R}_{l}\left(\xi_{i}\right)=\alpha_{t_{l}} u_{w_{l}}\left[\begin{array}{ll}
1 & 0
\end{array}\right]^{T}+\mathbf{A}_{l} \sum_{k=1}^{N} w_{k} \mathbf{H}^{p}\left(\mp \tau, \mp \xi_{k}\right) f_{l}\left(\xi_{k}, \xi_{i}\right)-\mathbf{H}^{p}\left(\mp \tau, \pm \xi_{i}\right)
$$


tal equação engloba de maneira unificada os problemas da dinâmica de gases rarefeitos propostos neste estudo.

Uma vez calculadas as soluções exatas, o próximo passo é determinar os $4 N$ coeficientes da Eq. 2.13. Para isso, é necessário substituir a Eq. 2.13 na Eq. 2.15 e obter um sistema de equações diferenciais ordinárias $4 N \times 4 N$.

Portanto, a partir das soluções tem-se que as grandezas físicas de interesse dadas pelo perfil de velocidade e perfil fluxo de calor são definidos, respectivamente por

$$
\begin{aligned}
u(\tau) & =A_{1}+B_{1} \varepsilon \tau+ \\
& +\sum_{j=2}^{2 N}\left[A_{j} e^{-\frac{\varepsilon(a+\tau)}{v_{j}}}+B_{j} e^{-\frac{\varepsilon(a-\tau)}{v_{j}}}\right] \sum_{k=1}^{N} w_{k} \Psi\left(\xi_{k}\right)\left[\begin{array}{l}
1 \\
0
\end{array}\right]^{T}\left[\Phi_{+}\left(v_{j}\right)+\Phi_{-}\left(v_{j}\right)\right]
\end{aligned}
$$

$\mathrm{e}$

$$
q(\tau)=\sum_{j=2}^{2 N}\left[A_{j} e^{-\frac{\varepsilon(a+\tau)}{v_{j}}}+B_{j} e^{-\frac{\varepsilon(a-\tau)}{v_{j}}}\right] \sum_{k=1}^{N} w_{k} \Psi\left(\xi_{k}\right)\left[\begin{array}{c}
\xi_{k}^{2}-\frac{1}{2} \\
2^{1 / 2}
\end{array}\right]^{T}\left[\Phi_{+}\left(v_{j}\right)+\Phi_{-}\left(v_{j}\right)\right]
$$

\section{ASPECTOS COMPUTACIONAIS E RESULTADOS NUMÉRICOS}

Inicialmente deve-se definir o esquema de quadratura juntamente com a versão analítica do ADO. Destaca-se que o aumento do número de pontos de quadratura oferecem uma melhor convergência numérica [2]. Para a modelagem apresentada, será utilizado 45 pontos de quadratura para obtenção de quatro dígitos significativos, um valor dentro do padrão utilizado na literatura que de modo geral, varia entre 30 a 80 [14, 18, 26, 33,34]. Além disso, neste caso, o tempo de processamento é irrelevante, ou seja, inferiores a 1 segundo para pontos de quadratura menores que 90. A fim de calcular integrais no intervalo $[1, \infty)$, usa-se a seguinte transformação não-linear $u(\xi)=e^{-\xi}$ para mapear os pontos $\xi$ no intervalo $[0, \infty)$ sob $u \in[0,1]$. Em seguida, utiliza-se o esquema de quadratura Gauss-Legendre [7] mapeado linearmente no intervalo $[0,1]$.

Os valores escolhidos para gerar os resultados numéricos das grandezas físicas são fornecidos na Tabela 2. Considera-se a grandeza física perfil de fluxo de calor e velocidade, levando em consideração as placas heterogêneas, ou seja, com coeficientes de acomodação tangencial e normal diferentes. Além disso, a largura do canal também define-se diferente a cada tabela e o parâmetro de livre caminho médio $(\varepsilon)$ oscila entre viscosidade $\left(\varepsilon_{p}\right)$ e condutividade térmica $\left(\varepsilon_{t}\right)$.

Destaca-se que para os casos dos Modelos BGK e $\mathbf{S}$ para $\varepsilon=\varepsilon_{p}$ a validação é realizado com os trabalhos [19] e [31], respectivamente ao considerar nos testes os coeficientes de acomodação iguais. Consequentemente, em virtude do modelo ser unificado, obtém-se a validação de resultados dos demais modelos abordados neste trabalho.

Ainda, a maioria dos trabalhos utilizam a simetria das superfícies, ou seja, o eixo $x$ no centro do canal já que as placas são homogêneas. Neste trabalho, propõe-se a utilização de coeficientes de acomodação diferentes e portanto a simetria das superfícies não ocorre. Neste sentido, afim de comparações, utiliza-se o recurso de translação do eixo. Dessa forma, o centro do canal para uma 
Tabela 2: Valores definidos para validação do modelo.

\begin{tabular}{cccccccc}
\hline Caso & $\alpha_{t_{1}}$ & $\alpha_{n_{1}}$ & $\alpha_{t_{2}}$ & $\alpha_{n_{2}}$ & $u_{w_{1}}$ & $u_{w_{2}}$ & $2 a^{\mathbf{1}}$ \\
\hline "A" & 0.25 & 0.50 & 0.75 & 0.25 & 1.0 & -2.0 & $1.0(\mathrm{X})$ \\
"B" & 0.50 & 0.75 & 0.25 & 0.25 & 0.0 & 1.0 & $2.0(\mathrm{X})$ \\
\hline
\end{tabular}
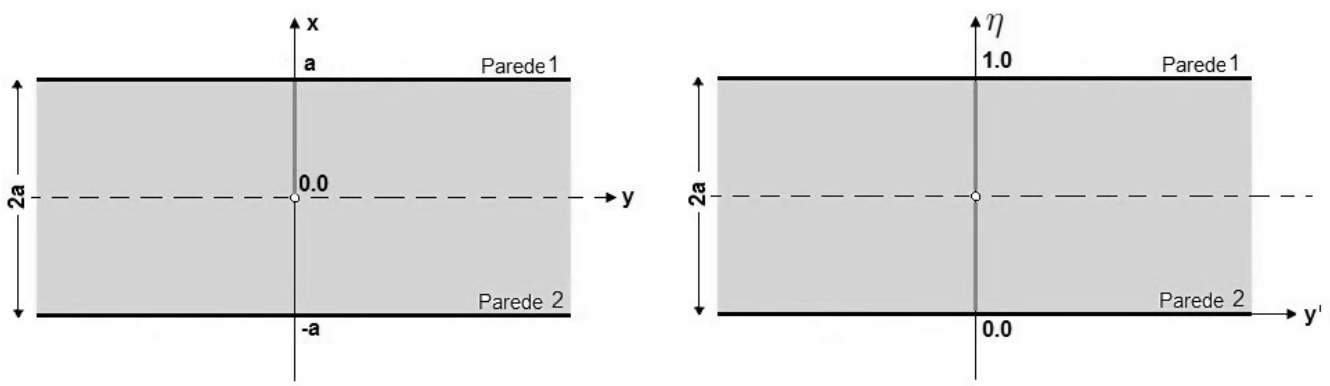

Figura 3: Disposição espacial das superfícies do canal: eixo transladado.

placa homogênea é dado no ponto $x=0.0$ e as extremidades em $a$ e $-a$, enquanto que para uma placa heterogênea o centro é dado em $\eta=0.5$ e as extremidades em $\eta=0.0$ e $\eta=1.0$. Essa localização espacial pode ser verificada na Figura 3.

A fim de validar a concordância dos modelos, será utilizado estatística não-paramétrica através da análise de variância de dois fatores de Friedman por postos. Para isso, define-se $\alpha=0.05$, o nível de significância, o procedimento no uso das análises de variância de dois fatores de Friedman por postos consiste em primeiramente considerar os escores em uma tabela de dupla entrada tendo $N$ linhas (sujeitos) e $k$ colunas (condições ou variáveis). Tem-se então, que $N=11$ é o número de posições no canal em cada um dos modelos cinéticos que totalizam $k=8$. Em seguida, será atribuído os postos aos dados de cada linha de 1 a $k$ e então somá-los em cada coluna. Tem-se também, a hipótese nula $\left(H_{0}\right)$ que define-se como que os modelos cinéticos não tem diferença entre si, enquanto que a hipótese alternativa $\left(H_{1}\right)$ é de que os modelos cinéticos diferem entre si significativamente.

\subsection{Problema de Poiseuille}

São tratados no Problema de Poiseuille os perfis de fluxo de calor $\left(q_{p}\right)$ e velocidade $\left(u_{p}\right)$. A velocidade das placas $\left(u_{w_{1}}\right.$ e $\left.u_{w_{2}}\right)$ será desconsiderada neste problema.

Nas Tabelas 3 e 4, são apresentados os valores numéricos para perfil fluxo de calor, no qual observa-se inicialmente que não ocorre simetria entre as fronteiras superiores e inferiores já que trata-se de placas heterogêneas. Analisando numericamente os resultados, tem-se que o Modelo GJ tem maior valor absoluto ao comparar com os demais modelos abordados. O Modelo BGK considerando $\varepsilon_{p}$ e Modelo MRS para $\varepsilon_{t}$ tem menor valor em módulo. Nesse sentido, tem-se que o modelo cinético que mais aproxima seus resultados numéricos da ELB, isto é, que comparado 
Tabela 3: Problema de Poiseuille: $q_{p}(-a+2 a \eta)$ para Caso "A" $\operatorname{com} \varepsilon=\varepsilon_{p}$.

\begin{tabular}{cccccc}
\hline$\eta$ & BGK & S & GJ & MRS & ELB [14] \\
\hline 0.0 & $1.2476(-1)$ & $1.6431(-1)$ & $1.6755(-1)$ & $1.6094(-1)$ & $9.5650(-2)$ \\
0.2 & $1.8637(-1)$ & $2.3631(-1)$ & $2.5250(-1)$ & $2.1695(-1)$ & $1.5862(-1)$ \\
0.4 & $1.9851(-1)$ & $2.5121(-1)$ & $2.7000(-1)$ & $2.2957(-1)$ & $1.7148(-1)$ \\
0.6 & $1.8763(-1)$ & $2.3752(-1)$ & $2.5262(-1)$ & $2.2168(-1)$ & $1.6197(-1)$ \\
0.8 & $1.5069(-1)$ & $1.9228(-1)$ & $1.9668(-1)$ & $1.9157(-1)$ & $1.2678(-1)$ \\
1.0 & $4.1982(-2)$ & $6.8671(-2)$ & $4.6411(-2)$ & $1.0616(-1)$ & $1.9727(-2)$ \\
\hline
\end{tabular}

Tabela 4: Problema de Poiseuille: $q_{p}(-a+2 a \eta)$ para Caso "B" com $\varepsilon=\varepsilon_{t}$.

\begin{tabular}{cccccc}
\hline$\eta$ & BGK & S & GJ & MRS & ELB [14] \\
\hline 0.0 & $2.7700(-1)$ & $3.0219(-1)$ & $3.3321(-1)$ & $2.5602(-1)$ & $-1.9451(-1)$ \\
0.2 & $2.7914(-1)$ & $3.0327(-1)$ & $3.3317(-1)$ & $2.5784(-1)$ & $1.6753(-1)$ \\
0.4 & $2.7155(-1)$ & $2.9214(-1)$ & $3.1799(-1)$ & $2.5176(-1)$ & $2.1371(-1)$ \\
0.6 & $2.5203(-1)$ & $2.6589(-1)$ & $2.8389(-1)$ & $2.3592(-1)$ & $2.1962(-1)$ \\
0.8 & $2.1376(-1)$ & $2.1587(-1)$ & $2.1989(-1)$ & $2.0459(-1)$ & $1.8979(-1)$ \\
1.0 & $1.1234(-1)$ & $8.9549(-2)$ & $6.0825(-2)$ & $1.2324(-1)$ & $5.2614(-2)$ \\
\hline
\end{tabular}

aos modelos cinéticos, é o que apresenta os menores valores absolutos, ou seja, é o Modelo BGK para $\varepsilon_{p}$ e Modelo MRS para $\varepsilon_{t}$, sendo que este último se compararmos ambos os modelos, é o que possui menor valor absoluto, o que significa que é o que mais se aproxima dos valores da ELB.

Aplicando a matemática estatística não-paramétrica, mostra-se a Tabela 5 que apresenta o resultado para este caso. A hipótese nula de que não havia diferença entre os modelos cinéticos ${ }^{1}$ foi rejeitada devido $p=0.0$, e portanto, conclui-se que há uma diferença entre os modelos cinéticos. Contudo, mesmo concluindo que há uma diferença, ainda não sabemos se há uma diferença entre os modelos cinéticos e a ELB. Para saber se ocorrem diferenças, devemos determinar as comparações múltiplas para todos os modelos cinéticos e a ELB.

Primeiramente, vamos trabalhar com $q_{p}(-a+2 a \eta)$ para o Caso "B" no qual iremos determinar a diferença entre os modelos cinéticos e a ELB. Utilizando estatística conclui-se que qualquer diferença que exceda $c=36.42$ indicará uma diferença significante entre o modelo cinético e a ELB. Como somente as diferenças da $R_{\mathbf{E L B}}$ com o $R_{\mathbf{S}_{\varepsilon_{\mathbf{p}}}}(c=45)$ e $R_{\mathbf{G} \mathbf{J}_{\varepsilon_{\mathbf{p}}}}(c=51)$ excedem a diferença crítica, concluímos que somente a diferença entre os Modelos $\mathbf{S}$ e $\mathbf{G J}$ para $\varepsilon=\varepsilon_{p}$ com a ELB são significantes. Note que $R_{\mathbf{M R S}}$, apesar de grande $(c=35)$, não é uma magnitude grande o suficiente para nos permitir concluir que Modelo MRS para $\varepsilon=\varepsilon_{p}$ e ELB são diferentes quando usamos o nível de significância que escolhemos.

\footnotetext{
${ }^{1}$ Quando refere-se aos modelos cinéticos das hipóteses nula, está sendo incluído, nestes casos, a ELB. Isso significa que não está sendo afirmado que há diferença da ELB com os demais modelos, mas sim que algum dos modelos, podendo ser a ELB, é diferente dos demais.
} 
Tabela 5: Problema de Poiseuille: Soma dos Postos $\left(R_{j}\right)$ para $q_{p}(-a+2 a \eta)$.

\begin{tabular}{lcccccccc}
\hline & BGK & \multicolumn{2}{c}{ S } & \multicolumn{2}{c}{ GJ } & \multicolumn{2}{c}{ MRS } & ELB [14] \\
\cline { 2 - 9 } & $\varepsilon_{p}=\varepsilon_{t}$ & $\varepsilon_{p}$ & $\varepsilon_{t}$ & $\varepsilon_{p}$ & $\varepsilon_{t}$ & $\varepsilon_{p}$ & $\varepsilon_{t}$ & \\
\hline Caso "A" & 35 & 76 & 40 & 82 & 58 & 66 & 28 & 11 \\
Caso "B" & 36 & 77 & 45 & 85 & 52 & 63 & 27 & 11 \\
\hline
\end{tabular}

Para a análise do $q_{p}(-a+2 a \eta)$ para o Caso "B" a partir da Tabela 5 obtém-se que as diferenças dos modelos cinéticos $\left|R_{\mathbf{S}_{\varepsilon_{\mathbf{p}}}}-R_{\mathbf{E L B}}\right|=|77-31|=46>36.42$ e $\left|R_{\mathbf{G J}_{\boldsymbol{\varepsilon}_{\mathbf{p}}}}-R_{\mathbf{E L B}}\right|=|85-31|=$ $54>36.42$ são os que excedem o valor de $c$. Em virtude disso, a diferença dos Modelos $\mathbf{S}$ e GJ para $\varepsilon=\varepsilon_{p}$ com a ELB é significante.
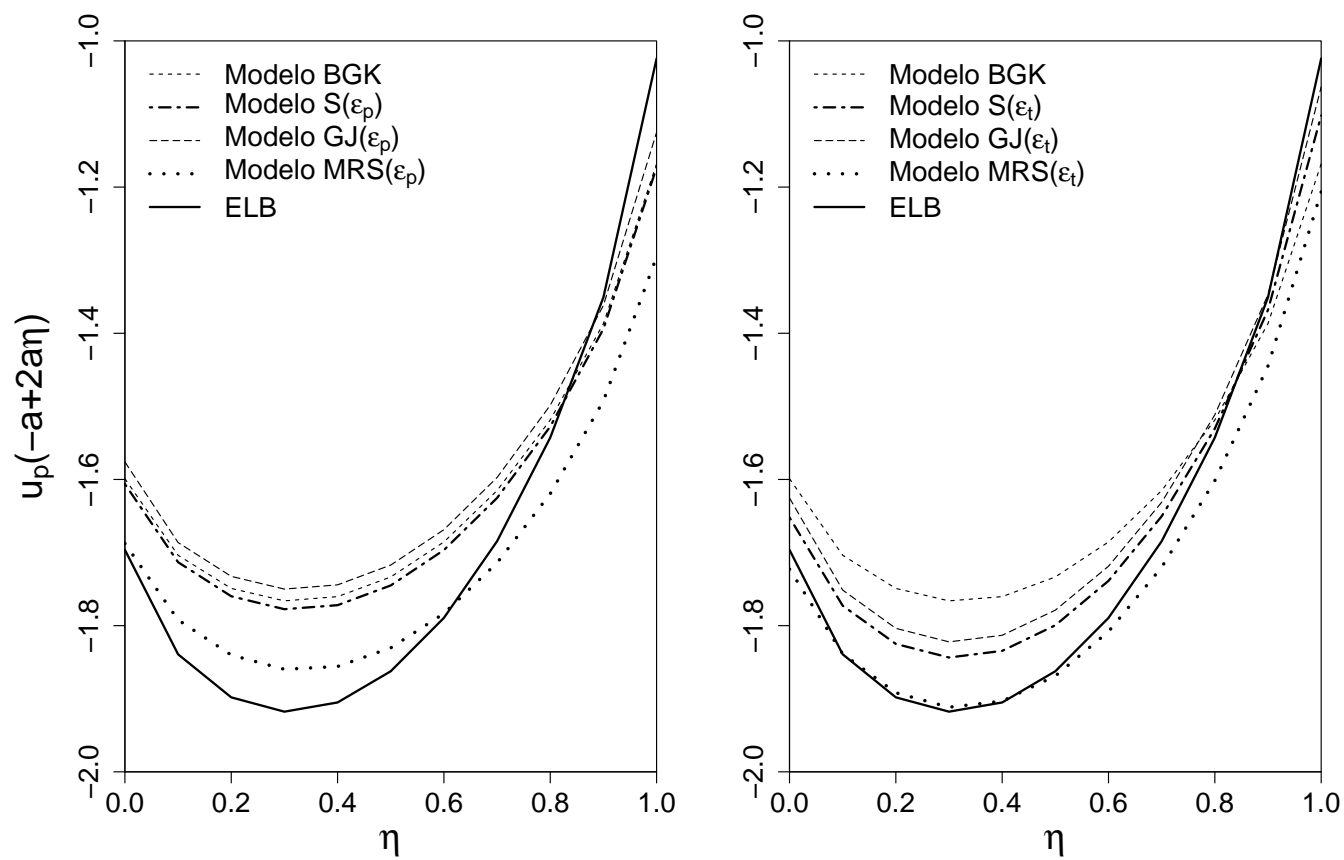

Figura 4: Problema de Poiseuille: $u_{p}(-a+2 a \eta)$ para Caso “A”.

Ao analisar os perfis de velocidade gerou-se a Figura 4, nas quais pode-se comparar visualmente os modelos cinéticos abordados neste trabalho. Inicialmente observa-se que devido as propriedades assimétrica das paredes a intensidade do fluxo não esta centralizado no centro, com maior tendência de deslocamento na placa superior $(\eta=1)$ onde os coeficientes de acomodação de energia cinética devido a componente normal da velocidade são mais altos. Destaca-se, neste caso, que a transferência de massa se desloca de maneira mais rápida através do perfil de velocidade em relação ao perfil de calor. 
Nota-se ainda que, utilizando o parâmetro $\varepsilon=\varepsilon_{t}$ os perfis estão mais próximos do que com $\varepsilon=\varepsilon_{p}$. Mas isso não auxilia no aumento de concordância do Modelo MRS que diverge em até um dígito dos demais modelos abordados. Contudo, visualmente, observa-se que o Modelo MRS com $\varepsilon_{t}$ dentre os modelos cinéticos é o que mais se aproxima dos valores da ELB.

\subsection{Problema Creep Térmico}

Serão tratados no Problema Creep Térmico os perfis de fluxo de calor $\left(q_{t}\right)$ e velocidade $\left(u_{t}\right)$. A velocidade das placas $\left(u_{w_{1}}\right.$ e $\left.u_{w_{2}}\right)$ será desconsiderada neste problema.

Tabela 6: Problema Creep Térmico: $q_{t}(-a+2 a \eta)$ para Caso "A".

\begin{tabular}{ccccccccc}
\hline$\eta$ & \multicolumn{1}{c}{ BGK } & \multicolumn{2}{c}{ S } & \multicolumn{2}{c}{ GJ } & \multicolumn{2}{c}{ MRS } & ELB [14] \\
\cline { 2 - 9 } & $\varepsilon_{p}=\varepsilon_{t}$ & $\varepsilon=\varepsilon_{p}$ & $\varepsilon=\varepsilon_{t}$ & $\varepsilon=\varepsilon_{p}$ & $\varepsilon=\varepsilon_{t}$ & $\varepsilon=\varepsilon_{p}$ & $\varepsilon=\varepsilon_{t}$ & \\
\hline 0.0 & -0.6862 & -0.8715 & -0.6685 & -0.8490 & -0.6481 & -0.9176 & -0.4956 & -0.4956 \\
0.2 & -0.8291 & -1.0585 & -0.8326 & -1.0640 & -0.8419 & -1.0706 & -0.8304 & -0.6539 \\
0.4 & -0.8600 & -1.1010 & -0.8687 & -1.1138 & -0.8858 & -1.1063 & -0.8581 & -0.6870 \\
0.6 & -0.8425 & -1.0754 & -0.8489 & -1.0845 & -0.8641 & -1.0882 & -0.8432 & -0.6733 \\
0.8 & -0.7708 & -0.9749 & -0.7661 & -0.9683 & -0.7684 & -1.0122 & -0.7810 & -0.6057 \\
1.0 & -0.5475 & -0.6858 & -0.5069 & -0.6330 & -0.4658 & -0.7917 & -0.5876 & -0.3590 \\
\hline
\end{tabular}

A Tabela 6 mostra os resultados numéricos para perfil fluxo de calor no Caso "A". Destaca-se que os maiores valores absolutos estão no centro do canal em termos de condutividade térmica. Nota-se que, para $\varepsilon=\varepsilon_{p}$, o modelo cinético que possui o maior valor absoluto está no Modelo GJ quando $\eta=0.4$ mas, o modelo que possui a maior quantidade de valores com maior valor absoluto encontra-se no Modelo MRS. Já o modelo que possui o menor valor absoluto é o Modelo BGK e consequentemente é o que mais se aproxima dos valores da ELB. Por outro lado, a análise para $\varepsilon=\varepsilon_{t}$ é contrária pois os valores oscilam entre dois modelos cinéticos, já que os maiores e menores valores absoluto estão nos Modelos GJ e MRS respectivamente. Para o Modelo GJ, os maiores valores em módulo estão na parte central do canal enquanto que os menores valores estão nas extremidades e para o Modelo MRS ocorre o inverso.

Partindo da matemática estatística, considera-se o nível de significância de 5\%, para encontrar os valores da soma dos postos. Tem-se que $p=0.0$, ou seja, a hipótese nula foi rejeitada e isso nos faz interpretar que há uma diferença entre os modelos cinéticos comparados entre si. Contudo, ainda não é possível afirmar se os modelos diferem da ELB. Para resolver esta questão, determina-se as comparações múltiplas para todos os modelos cinéticos e a ELB. Ao desenvolver, conclui-se que os Modelos S, GJ e MRS para $\varepsilon=\varepsilon_{p}$ comparados com a ELB possuem diferença significativa e portanto pode-se rejeitar os três modelos cinéticos. Ainda com o auxílio gráfico, conclui-se então que para o perfil fluxo de calor que o modelo que melhor se aproxima da ELB é o Modelo MRS $\operatorname{com} \varepsilon=\varepsilon_{t}$.

Para analisar o perfil de velocidade apresenta-se a Figura 5. Nota-se que as extremidades das placas tem melhor convergência entre os modelos do que o centro. Além disso, é possível observar 
que os modelos tem maior convergência entre si se considerarmos o mesmo livre caminho médio, sendo que o Modelo BGK mostra uma tendência de se agrupar ao parâmetro de condutividade térmica apesar de não apresentar distinção dos valores neste modelo. O perfil de velocidade plotado detecta facilmente que o Modelo MRS para $\varepsilon=\varepsilon_{t}$ é o que mais está se aproximando da ELB com exceção da extremidade da placa inferior no qual Modelo BGK apresenta melhor comportamento.

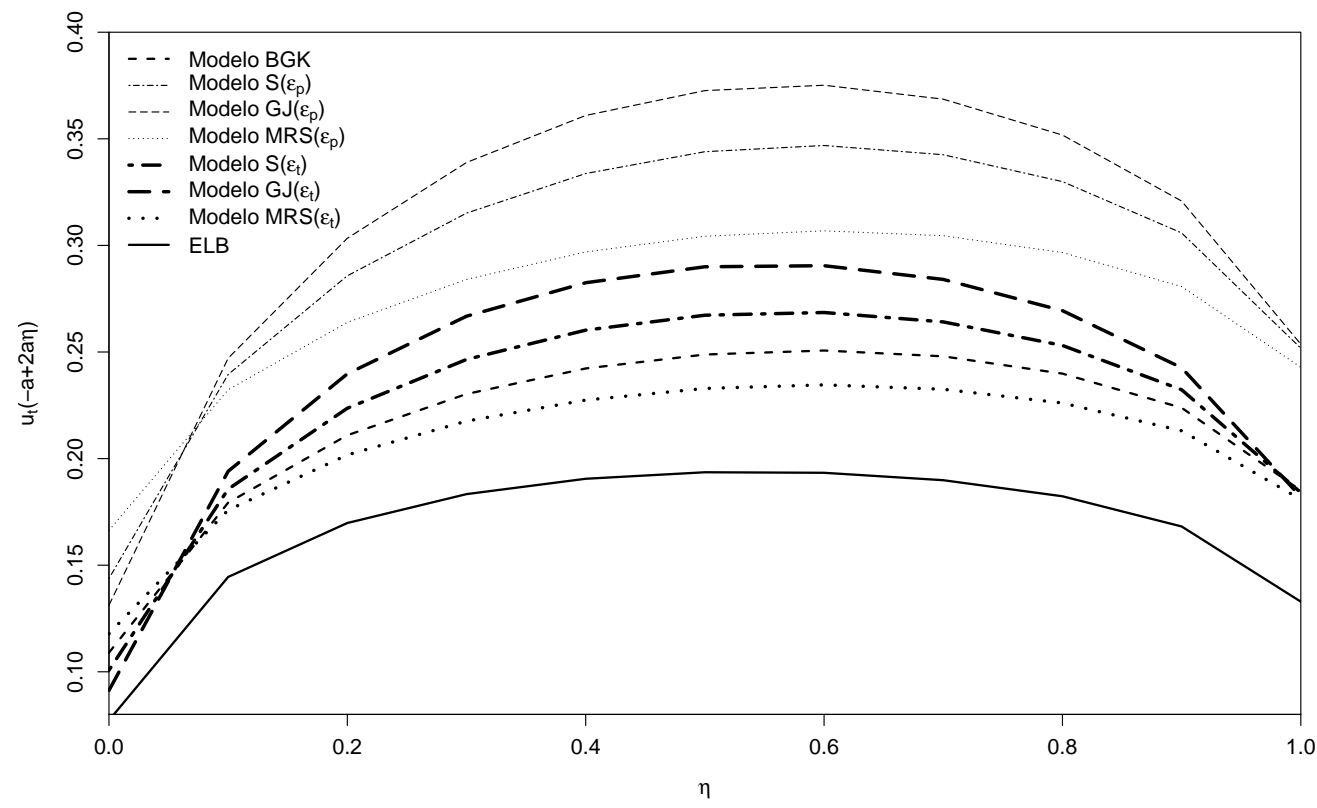

Figura 5: Problema Creep Térmico: $u_{t}(-a+2 a \eta)$ para Caso "A".

Novamente, o teste estatístico matemático rejeita os seguintes modelos cinéticos: $\mathbf{S}$ e MRS para $\varepsilon=\varepsilon_{p}$, além disso, recusa-se o Modelo GJ para $\varepsilon_{p}$ e $\varepsilon_{t}$. Em consequência, temos assim como para o Problema de Poiseuille, tanto para o perfil fluxo de calor como para o perfil de velocidade, que o Modelo MRS para $\varepsilon=\varepsilon_{t}$ para o Problema Creep Térmico é o modelo que mais se aproxima da ELB.

\subsection{Problema de Couette}

Ao analisar o Problema de Couette, o modelo foi validado através dos resultados numéricos do trabalho [19] para o Modelo BGK, restritos inicialmente, apenas para placas iguais e consequentemente, em virtude do modelo ser genérico, para placas heterogêneas. Como demais problemas, será dado ênfase ao perfil fluxo de calor $\left(q_{c}\right)$ e velocidade $\left(u_{c}\right)$ ao Problema de Couette. 
Tabela 7: Problema de Couette: $q_{c}(-a+2 a \eta)$ para Caso "B" na ordem de $10^{-3}$.

\begin{tabular}{crrrrrrrr}
\hline$\eta$ & \multicolumn{1}{c}{ BGK } & \multicolumn{2}{c}{ S } & \multicolumn{2}{c}{ GJ } & \multicolumn{2}{c}{ MRS } & ELB [14] \\
\cline { 2 - 9 } & $\varepsilon_{p}=\varepsilon_{t}$ & $\varepsilon=\varepsilon_{p}$ & $\varepsilon=\varepsilon_{t}$ & $\varepsilon=\varepsilon_{p}$ & $\varepsilon=\varepsilon_{t}$ & $\varepsilon=\varepsilon_{p}$ & $\varepsilon=\varepsilon_{t}$ & \\
\hline 0.0 & 13.134 & 13.985 & 14.144 & 16.427 & 16.315 & 9.784 & 10.117 & 8.101 \\
0.2 & 4.113 & 4.793 & 4.488 & 5.761 & 5.237 & 3.339 & 3.155 & 2.567 \\
0.4 & 0.348 & 0.163 & 0.449 & 0.292 & 0.633 & 0.155 & 0.262 & 0.400 \\
0.6 & -2.980 & -4.112 & -3.161 & -4.817 & -3.455 & -2.716 & -2.270 & -1.421 \\
0.8 & -7.410 & -9.494 & -8.063 & -1.135 & -9.251 & -6.273 & -5.596 & -4.294 \\
1.0 & -19.226 & -21.501 & -21.243 & -25.888 & -25.133 & -14.153 & -14.399 & -12.114 \\
\hline
\end{tabular}

A partir do Caso "B", tem-se a Tabela 7 para o perfil fluxo de calor, no qual o deslocamento da vazão ocorrerá apenas pelo movimento gerado pela velocidade de uma placa, ou seja, uma das paredes ficará em repouso. Observa-se que os maiores valores absolutos são apresentados no Modelo GJ enquanto que os menores valores absolutos para o Modelo MRS, ambos considerando $\varepsilon=\varepsilon_{t}$ e portanto esse modelo é o que esta em maior concordância com a ELB já que a mesma apresenta os menores valores numéricos. Tal conclusão pode ser confirmada ao analisar a Figura 6 no qual é possível observar uma boa concordância entre os modelos cinéticos para o perfil fluxo de calor. Além disso, em uma análise mais detalhada no intervalo $0.6<\eta<0.9$ nota-se que o Modelo MRS para $\varepsilon_{t}$ é o mais próximo ao perfil da ELB.
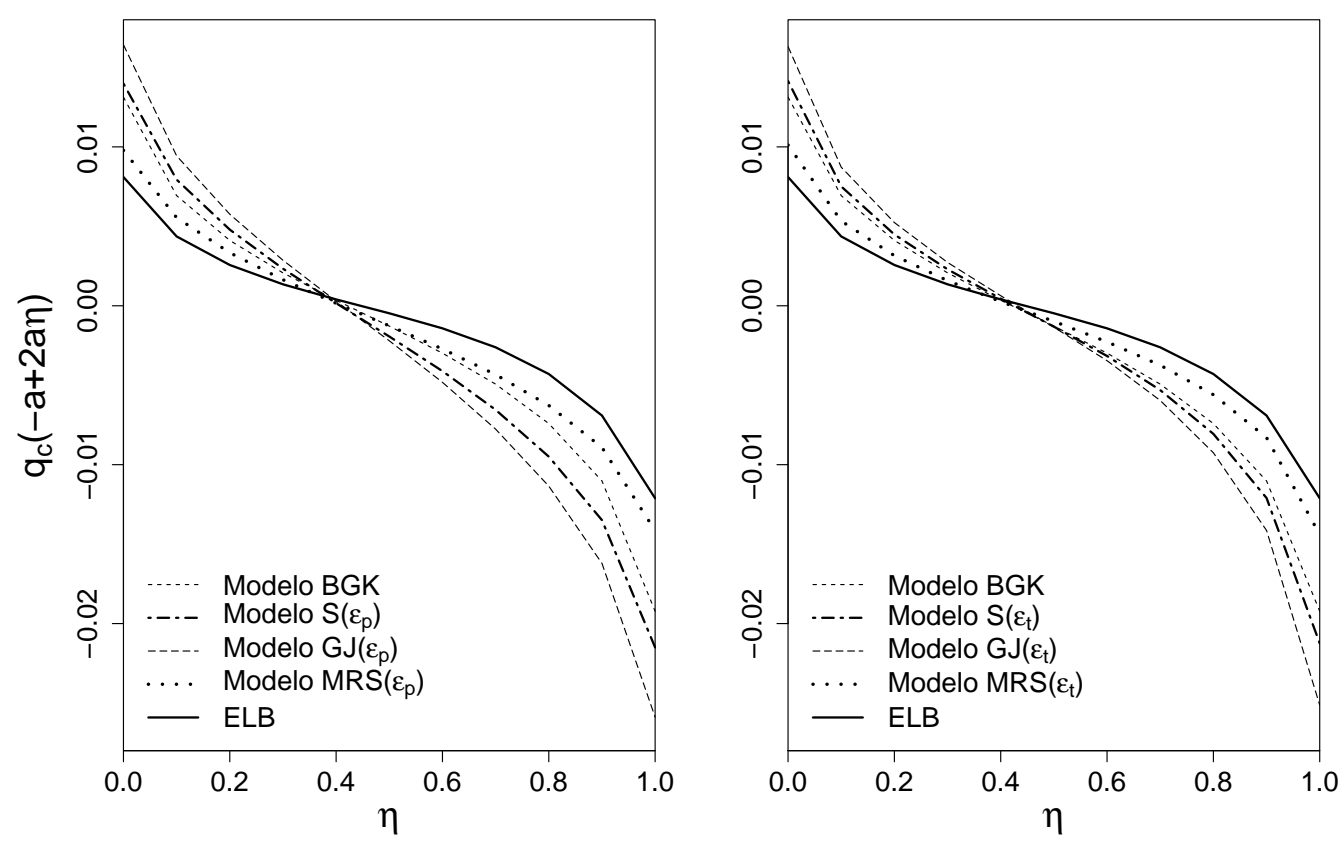

Figura 6: Problema de Couette: $q_{c}(-a+2 a \eta)$ para Caso "B". 
Além disso, da Figura 6 é possível observar que o perfil do fluxo de calor tende a ter uma simetria que se desloca do perfil negativo para o positivo já que a placa superior está em repouso, causando o maior intensidade de calor perto da prancha móvel. Ao considerar o caminho livre médio, notase que os modelos são congruentes entre si, com pouca divergência na definição do parâmetro tendendo a um melhor desempenho da viscosidade.

Baseado na estatística não paramétrica é possível definir com maior clareza a convergência entre os modelos. Para o Caso "A" foi obtido $p=0.71524>0.05$ e para o Caso "B", $p=0.77628>$ 0.05 , ou seja, como o valor do posto é maior do que o nível de significância, a hipótese nula não é rejeitada e isso implica que os modelos cinéticos não diferem entre si, o que confirma a concordância visualizada na Figura 6. Portanto, o Modelo MRS para $\varepsilon=\varepsilon_{t}$ é considerado o modelo mais preciso para o problema de Couette no perfil fluxo de calor.

Para a grandeza física perfil de velocidade apresenta-se a Tabela 8. Nela é possível destacar alguns resultados diferentes aos obtidos nos problemas anteriores. Para os valores $\varepsilon=\varepsilon_{t}$ tem-se que o Modelo MRS é o de maior valor absoluto e o Modelo GJ é o que possui menor valor absoluto, já para $\varepsilon=\varepsilon_{p}$, o Modelo BGK maior valor absoluto e o Modelo GJ é o que possui menor valor absoluto. Portanto, o modelo cinético que melhor se aproxima da ELB é o Modelo GJ $\operatorname{com} \varepsilon=\varepsilon_{t}$. Essa conclusão pode ser verificada na Figura 7.

Tabela 8: Problema de Couette: $u_{c}(-a+2 a \eta)$ para Caso “A”.

\begin{tabular}{ccccccccc}
\hline$\eta$ & \multicolumn{1}{c}{ BGK } & \multicolumn{2}{c}{ S } & \multicolumn{2}{c}{ GJ } & \multicolumn{2}{c}{ MRS } & ELB [14] \\
\cline { 2 - 9 } & $\varepsilon_{p}=\varepsilon_{t}$ & $\varepsilon=\varepsilon_{p}$ & $\varepsilon=\varepsilon_{t}$ & $\varepsilon=\varepsilon_{p}$ & $\varepsilon=\varepsilon_{t}$ & $\varepsilon=\varepsilon_{p}$ & $\varepsilon=\varepsilon_{t}$ & \\
\hline 0.0 & -1.0296 & -1.0286 & -0.9065 & -1.0120 & -0.8929 & -1.0623 & -0.9371 & -0.7703 \\
0.2 & -1.1532 & -1.1522 & -1.0661 & -1.1468 & -1.0641 & -1.1671 & -1.0766 & -0.9737 \\
0.4 & -1.2496 & -1.2490 & -1.1906 & -1.2453 & -1.1887 & -1.2582 & -1.1975 & -1.1261 \\
0.6 & -1.3420 & -1.3420 & -1.3103 & -1.3388 & -1.3073 & -1.3472 & -1.3156 & -1.2724 \\
0.8 & -1.4376 & -1.4380 & -1.4338 & -1.4361 & -1.4304 & -1.4380 & -1.4362 & -1.4233 \\
1.0 & -1.5588 & -1.5592 & -1.5895 & -1.5670 & -1.5957 & -1.5420 & -1.5743 & -1.6204 \\
\hline
\end{tabular}

Nota-se que o Modelo MRS para $\varepsilon_{t}$ que vinha se destacando como modelo cinético que mais se aproxima da ELB para os problemas proposto, no caso do perfil velocidade no Problema de Couette, se mostrou uma das opções menos adequadas.

Ao analisar os resultados obtidos para perfil de velocidade na Tabela 9, confirma-se que o Modelo GJ e Modelo MRS apresentam valores significativamente menores em módulo do que os demais modelos para alguns pontos $\eta$, considerando tanto o parâmetro de viscosidade como de condutividade térmica, sendo que estes modelos oscilam em quem tem o menor valor absoluto. Dessa forma, o Modelo GJ e MRS são os que mais se aproximam da ELB, onde destaca-se nas extremidades o Modelo GJ e no centro do canal o Modelo MRS, tal resultado fica evidente na Figura 7. Ainda, no perfil de velocidade, é possível observar que no Caso "A”, todo o perfil está em uma área negativa e no Caso "B" em uma área positiva. Essa percepção sugere que a velocidade das placas está dominando o perfil de velocidade. Além disso, tem-se que para os valores 
Tabela 9: Problema de Couette: $u_{c}(-a+2 a \eta)$ para Caso “B”.

\begin{tabular}{ccccccccc}
\hline$\eta$ & BGK & \multicolumn{2}{c}{ S } & \multicolumn{2}{c}{ GJ } & \multicolumn{2}{c}{ MRS } & ELB [14] \\
\cline { 2 - 9 } & $\varepsilon=\varepsilon_{p}=\varepsilon_{t}$ & $\varepsilon=\varepsilon_{p}$ & $\varepsilon=\varepsilon_{t}$ & $\varepsilon=\varepsilon_{p}$ & $\varepsilon=\varepsilon_{t}$ & $\varepsilon=\varepsilon_{p}$ & $\varepsilon=\varepsilon_{t}$ & \\
\hline 0.0 & 0.2224 & 0.2222 & 0.2025 & 0.2204 & 0.2013 & 0.2269 & 0.2060 & 0.1821 \\
0.2 & 0.2763 & 0.2761 & 0.2707 & 0.2768 & 0.2718 & 0.2761 & 0.2698 & 0.2660 \\
0.4 & 0.3197 & 0.3198 & 0.3265 & 0.3199 & 0.3266 & 0.3195 & 0.3263 & 0.3345 \\
0.6 & 0.3623 & 0.3626 & 0.3812 & 0.3621 & 0.3804 & 0.3624 & 0.3821 & 0.4019 \\
0.8 & 0.4070 & 0.4076 & 0.4384 & 0.4068 & 0.4368 & 0.4066 & 0.4395 & 0.4713 \\
1.0 & 0.4665 & 0.4671 & 0.5133 & 0.4705 & 0.5156 & 0.4587 & 0.5069 & 0.5625 \\
\hline
\end{tabular}
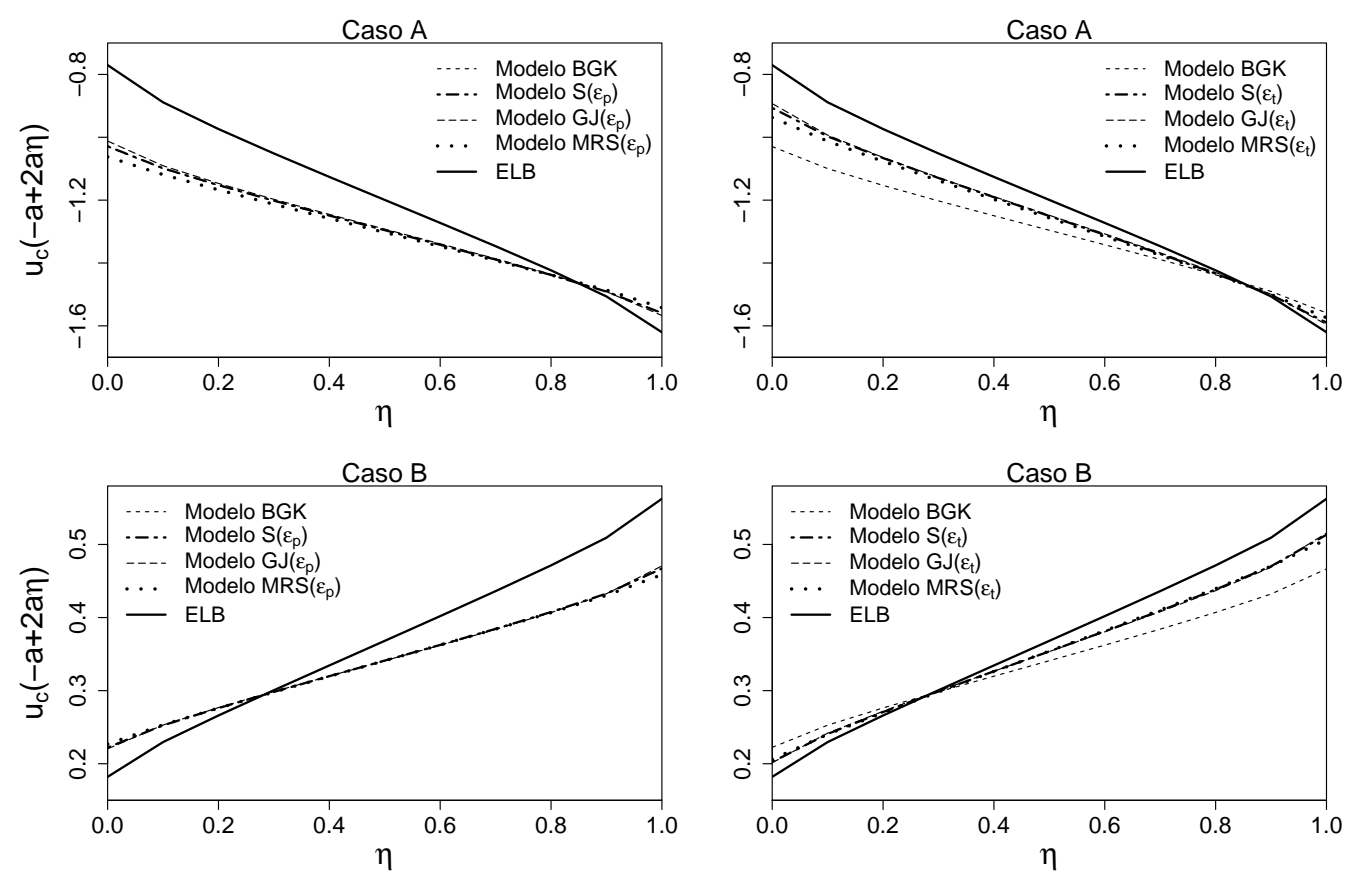

Figura 7: Problema de Couette: $u_{c}(-a+2 a \eta)$ para Caso "A" e "B".

de $\varepsilon_{p}$ que o modelo com maiores valores em módulo é o Modelo MRS, enquanto que para $\varepsilon_{t}$ é o Modelo BGK. Confirma-se então que, o Modelo MRS, que nos demais problemas era o modelo que mais se aproxima da ELB, neste caso é o de menor concordância.

Contudo, ao considerar matemática estatística tem-se do Caso "B" que $p=0.07768>0.05$, ou seja, não tem diferença entre os modelos cinéticos. Já para o Caso "A" tem-se que $p=0.00005$ e como é menor que o nível de significância $\alpha=0.05$, pode-se rejeitar a hipótese nula e isso implica que há diferença entre os modelos cinéticos. Trabalha-se inicialmente determinando-se a diferença entre os modelos cinéticos e a ELB. Observa-se que três modelos cinéticos excedem o valor de $c$ e então indicam uma diferença significativa entre o modelo e a ELB. Como somente 
as diferenças da $R_{\mathbf{E L B}}$ com o $R_{\mathbf{B G K}}(c=39.5), R_{\mathbf{S}_{\boldsymbol{q}_{\mathbf{p}}}}(c=36)$ e $R_{\mathbf{M R S}_{\varepsilon_{\mathbf{p}}}}(c=25.5)$ excedem a diferença crítica, conclui-se que somente a diferença entre as condições Modelos BGK, $\mathbf{S}$ e MRS para $\varepsilon=\varepsilon_{p}$ com a ELB são significantes.

Quando analisa-se apenas as tabelas e as figuras para perfil de velocidade conclui-se que o Modelo GJ para $\varepsilon=\varepsilon_{t}$ é o que melhor se aproxima dos valores numéricos da ELB. Contudo, com o resultado estatístico conclui-se que para o Caso "B" os modelos não diferem, e que para o Caso "A" alguns modelos diferem, mas que o Modelo S com $\varepsilon_{t}$, Modelos GJ e MRS com $\varepsilon_{t}$ não diferem significativamente entre si. Em virtude disso, e como para o perfil fluxo de calor o modelo cinético escolhido é o Modelo MRS para $\varepsilon=\varepsilon_{t}$, pode-se concluir que o Modelo MRS para $\varepsilon_{t}$ é uma escolha adequada tanto para o perfil fluxo de calor como para o perfil de velocidade.

\section{CONCLUSÃo}

Neste artigo, apresenta-se resultados numéricos para perfil fluxo de calor e perfil de velocidade para três problemas da dinâmica de gases rarefeitos, que são: Problema de Poiseuille, Creep Térmico e Couette que foram obtidos pelo método ADO. Esse método mostrou-se de grande eficiência computacional, já que possibilitou a resolução de uma variedade de problemas, principalmente quando trata-se o desenvolvimento unificado das equações modelo em tempo de processamento irrelevante para o número de dígitos significativos comparados com a literatura [14, 18, 26, 33, 34].

De modo geral, os quatro modelos cinéticos analisados estatisticamente mostraram-se uma boa alternativa, conforme observado na Tabela 10, que mostra o resumo dos resultados obtidos no qual indica com " $x$ " os modelos rejeitados nos respectivos testes realizados.

Tabela 10: Modelos Cinéticos Rejeitados para cada quantidade física de interesse.

\begin{tabular}{cccccccc}
\hline & \multicolumn{1}{c}{$\mathbf{B G K}$} & \multicolumn{2}{c}{$\mathbf{S}$} & \multicolumn{2}{c}{ GJ } & \multicolumn{2}{c}{ MRS } \\
\cline { 2 - 8 } & $\varepsilon=\varepsilon_{p}=\varepsilon_{t}$ & $\varepsilon=\varepsilon_{p}$ & $\varepsilon=\varepsilon_{t}$ & $\varepsilon=\varepsilon_{p}$ & $\varepsilon=\varepsilon_{t}$ & $\varepsilon=\varepsilon_{p}$ & $\varepsilon=\varepsilon_{t}$ \\
\hline $\mathbf{q}_{\mathbf{p}}$ & - & $\mathrm{x}$ & - & $\mathrm{x}$ & - & - & - \\
$\mathbf{u}_{\mathbf{p}}$ & $\mathrm{x}$ & - & - & $\mathrm{x}$ & - & - & - \\
$\mathbf{q}_{\mathbf{t}}$ & - & $\mathrm{x}$ & - & $\mathrm{x}$ & - & $\mathrm{x}$ & - \\
$\mathbf{u}_{\mathbf{t}}$ & - & $\mathrm{x}$ & - & $\mathrm{x}$ & $\mathrm{x}$ & $\mathrm{x}$ & - \\
$\mathbf{q}_{\mathbf{c}}$ & - & - & - & - & - & - & - \\
$\mathbf{u}_{\mathbf{c}}$ & $\mathrm{x}$ & $\mathrm{x}$ & - & - & - & $\mathrm{x}$ & - \\
\hline
\end{tabular}

Logo, tem-se que dois modelos cinéticos destacaram-se a partir da matemática estatística, são eles, Modelos $\mathbf{S}$ e MRS para $\varepsilon=\varepsilon_{t}$ que não foram rejeitados por nenhuma quantidade física e o Modelos $\mathbf{S}$ e o GJ para $\varepsilon_{p}$ como os modelo mais rejeitados. Portanto, o Modelo MRS com o parâmetro de livre caminho médio é o modelo que mais se aproxima da ELB, por outro lado, o Modelo GJ é o pior modelo. Ainda, na análise dos resultados através da matemática estatística foi analisado o comportamento de cada modelo em relação a ELB. Somente no Problema de Couette, no perfil fluxo de calor nenhum modelo foi rejeitado, ou seja, foi o problema no qual 
os modelos apresentaram maior concordância. Além disso, a influência do parâmetro $\varepsilon$ é significativo, ou seja, quando toma-se o parâmetro $\varepsilon=\varepsilon_{t}$, obtém-se resultados numéricos melhores quando comparados aos valores da ELB e também observou-se os modelos tinham uma maior concordância entre si quando utilizado o parâmetro de livre caminho médio para condutividade térmica, conforme Tabela 10, onde os modelos rejeitados eram os que consideraram $\varepsilon_{p}$.

Destaca-se ainda, que para o Problema de Poiseuille e Creep Térmico, o centro do canal é que possui os maiores valores numéricos absolutos se considerarmos o perfil fluxo de calor e perfil de velocidade. Já para o Problema de Couette, os maiores valores numéricos para os perfis ocorrem nas extremidades do canal, o que fisicamente é esperado já que o que provoca o fluxo de gás é o movimento das placas. Analisando o Problema de Couette para o perfil fluxo de calor tem-se que na proximidade do centro do canal o fluxo é praticamente nulo próxima ao centro do canal, sendo que aumenta conforme se aproxima das extremidades do canal. Quando analisa-se o perfil de velocidade este perfil não se anula no centro do canal mas sim, na metade superior do canal $(\eta=0.8)$ ou na metade inferior do canal $(\eta=0.3)$.

Além disso, neste trabalho, foram demostrados novos resultados numéricos. Salienta-se, os resultados gerados para o Problema de Couette para placas diferentes. Isso decorre em virtude do problema ser homogêneo, ou seja, do seu termo fonte ser igual a zero. Logo, este problema foi resolvido acrescentado um termo não homogêneo na condição de contorno de $\mathbf{C L}$, onde fisicamente isso é possível, bastando analisar que o fluxo de gás ocorre devido ao movimento das placas. O canal onde ocorre o fluxo de gás foi analisado de forma completa, isto é, foram obtidos resultados numéricos de uma extremidade a outra da superfície em virtude de considerar placas com propriedades físicas diferentes. Isso, nos trabalhos atuais não era necessário já que como as placas eram homogêneas elas eram simétricas e bastava apenas a análise da metade superior ou inferior do canal.

\begin{abstract}
The phenomena that involve the rarefied gas dynamics for gas flow in microchannels have been a source of study in several studies. The Boltzmann Equation describes such phenomena, and from its simplification comes the kinetic theory for the dynamics of rarefied gases. The kinetic models present reliable results and are analyzed in this study under an analytical version using the discrete ordinate method. Therefore, we explore a model closer to reality, comparing the numerical values generated by four kinetic models derived in a unified way from Boltzmann's Linearized Equation. In terms of viscosity and thermal conductivity, the models are compared based on non-parametric statistics for problems with rarefied gas dynamics resulting from the mass transfer movement. Also, the gas-surface interaction defined by the Cercignani-Lampis nucleus along a channel for heterogeneous plates is used.
\end{abstract}

Keywords: rarefied gas dynamics, analytical discrete ordinates method, kinetic model, Cercignani-Lampis scattering kernel. 


\section{REFERÊNCIAS}

[1] L. Barichello, M. Camargo, P. Rodrigues \& C. Siewert. Unified Solutions to Classical Flow Problems Based on the BGK Model. Zeitschrift für Angewandte Mathematik und Physik, 52 (2001), 517-534.

[2] L. Barichello \& C. Siewert. A discrete-ordinates solution for a non-grey model with complete frequency redistribution. Journal of Quantitative Spectroscopy and Radiative Transfer, 62 (1999), $665-675$.

[3] L. Barichello \& C. Siewert. Some Comments on Modeling de Linearized Boltzmann Equation. Journal of Quantitative Spectroscopy and Radiative Transfer, 77 (2003), 43-59.

[4] R. Benzi, S. Succi \& M. Vergassola. The lattice Boltzmann equation: theory and applications. Physics Reports, 222(3) (1992), 145-197.

[5] P. Bhatnagar, E. Gross \& M. Krook. A model for collision processes in gases. Physical Review, 94 (1954), 511-525.

[6] A. Bobylev, M. Bisi, M. Groppi, G. Spiga \& I. Potapenko. A general consistent BGK model for gas mixtures. Kinetic \& Related Models, 11(6) (2018), 1377.

[7] L. Burden \& D. Faires. "Numerical Analysis", volume I. Brooks/Cole Publishing, Boston (1997).

[8] C. Cercignani. "Mathematical Methods in Kinetic Theory", volume I. Plenum Press, New York (1969).

[9] C. Cercignani. "The Boltzmann Equation and its Applications”, volume I. Springer-Verlag (1988).

[10] C. Cercignani \& M. Lampis. Kinetic Models for Gas-Surface Interaction. Transport Theory Sthatistical Physics, 1 (1971), 101-114.

[11] R. Coelho \& M. Doria. Lattice Boltzmann method for semiclassical fluids. Computers \& Fluids, $\mathbf{1 6 5}$ (2018), 144-159.

[12] H. Ferziger \& G. Kaper. "Mathematical Theory of Transport Processes in Gases", volume I. NorthHolland Publishing Company, Amsterdam (1972).

[13] M. Garcia \& E. Siewert. The linearized Boltzmann equation: sound-wave propagation in a rarefied gas. Zeitschrift für Angewandte Mathematik und Physik, 57 (2006), 94-122.

[14] M. Garcia \& E. Siewert. The linearized Boltzmann equation with Cercignani-Lampis boundary conditions: Basic flow problems in a plane channel. Europen Journal of Mechanics B/Fluids, 28 (2009), 387-396.

[15] E. Gross \& E. Jackson. Kinetic models and the linearized Boltzmann equation. The Physics of Fluids, 2 (1959), 432-441.

[16] D. Kalempa. "Modelagem numérica de propagação de ondas em gases rarefeios". Ph.D. thesis, Tese de doutorado ao Programa de Pós-Graduação em Física, Setor de Ciências Exatas, UFPR, Curitiba, Brasil (2009). 
[17] D. Kalempa, F. Sharipov \& J. Silva. Sound waves in gaseous mixtures induced by vibro-thermal excitation at arbitrary rarefaction and sound frequency. Vacuum, 159 (2019), 82-98.

[18] R. Knackfuss \& I. Aseka. Problema Creep-Térmico na dinâmica de gases rarefeitos baseado no modelo BGK. TEMA, 13(1) (2012), 63-74.

[19] R. Knackfuss \& L. Barichello. Surface effects in rarefied gas dynamics: an analysis based on the Cercignani-Lampis boundary condition. Europen Journal of Mechanics B/Fluids, 25 (2006), 113129.

[20] L. Li, R. Mei \& J. Klausner. Lattice Boltzmann models for the convection-diffusion equation: D2Q5 vs D2Q9. International Journal of Heat and Mass Transfer, 108 (2017), 41-62.

[21] K. Loyalka \& H. Ferziger. Model dependence of the slip coefficient. The Physics of Fluids, 10 (1967), 1833-1938.

[22] K. Loyalka \& K. Hickey. A Plane Poiseuille flow: near continuum results for a rigid sphere gas. Physica A, 160 (1989), 395-408.

[23] J. Prolo Filho. "A Equação de Boltzmann e a Modelagem de Fluidos em Micro e Macroescala”. Ph.D. thesis, Dissertação de Mestrado do Programa de Pós graduação em Matemática Aplicada, UFRGS, Porto Alegre, Brasil (2007).

[24] C. Rosa \& R. Knackfuss. An unified formulation for the kinetics models in the Poiseuille flow based on the Cercignani-Lampis boundary condition. Books of ABSTRACTS - XXXII CILAMCE - Iberian Latin-American Congress on Computacional Methods in Engineering, (2011), 78.

[25] C. Scherer. "Modelos Cinéticos da Equação Linearizada de Boltzmann e um Problema de Transferência de Calor em Microescala". Ph.D. thesis, Dissertação de Mestrado do Programa de Pós graduação em Matemática Aplicada, UFRGS, Porto Alegre, Brasil (2005).

[26] C. Scherer \& L. Barichello. An analytical approach to the unified solution of kinetic equations in rarefied gas dynamics.I.Flow problems. Zeitschrift für Angewandte Mathematik und Physik, 60 (2009), $70-115$.

[27] F. Sharipov. "Method of investigation of rarefied gas flows", volume I. Nauka, Moscow (1974).

[28] F. Sharipov. Application of the Cercignani scattering kernel to calculation of rarefied gas flows.I.Plane Flow Between two Parallel Plates. Europen Journal of Mechanics B/Fluids, 21 (2002), 113-123.

[29] F. Sharipov. Application of the Cercignani scattering kernel to calculation of rarefied gas flows.II.Slip and jump coefficients. Europen Journal of Mechanics B/Fluids, 22 (2003), 133-143.

[30] F. Sharipov \& V. Seleznev. Data on internal rarefied gas flows. Journal Physical and Chemical Reference Data, 27 (1998), 657-706.

[31] C. Siewert. Generalized boundary conditions for the S-model kinetic equations basic to flow in a plane channel. Journal of Quantitative Spectroscopy and Radiative Transfer, 72 (2002), 75-88.

[32] C. Siewert. Two half-space problems based on a synthetic-kernel model of the linearized Boltzmann equation. Journal of Quantitative Spectroscopy and Radiative Transfer, 75 (2002), 21-38. 
[33] C. Siewert. Heat transfer and evaporation/condensation problems based on the linearized Boltzmann equation. European Journal of Mechanics-B/Fluids, 22(4) (2003), 391-408.

[34] C. Siewert. Viscous-Slip, Thermal-Slip and Temperature-Jump Coefficients as Defined by Linearized Boltzmann Equation and the Cercignani-Lampis Boundary Condition. Physical of Fluids, 15 (2003), 1696.

[35] W. Su, P. Wang, H. Liu \& L. Wu. Accurate and efficient computation of the Boltzmann equation for Couette flow: Influence of intermolecular potentials on Knudsen layer function and viscous slip coefficient. Journal of Computational Physics, 378 (2019), 573-590.

[36] G. Wick. Uber Ebene Diffusions Problem. Zeitschrift für Angewandte Mathematik und Physik, 120 (1943), 702-.

[37] M. Williams. A review of the rarefied gas dynamics theory associated with some classical problems in flow and heat transfer. Zeitschrift für Angewandte Mathematik und Physik, 52 (2001), 500-516.

[38] L. Wu, H. Liu, Y. Zhang \& J. Reese. Influence of intermolecular potentials on rarefied gas flows: Fast spectral solutions of the Boltzmann equation. Physics of Fluids, 27(8) (2015), 082002.

[39] Q. Zou, S. Hou \& G. Doolen. Analytical solutions of the lattice Boltzmann BGK model. Journal of Statistical Physics, 81 (1995), 319-334. 